\title{
EL PROCESO DE DIFUSIÓN, PARA VARIEDADES MEJORADAS DE Phaseolus vulgaris L. EN GUATEMALA1
}

\author{
Marco Baltensweiler ${ }^{2}$
}

\begin{abstract}
RESUMEN
El proceso de difusión para variedades mejoradas de Phaseolus vulgaris L. en Guatemala. Se ha descubierto una función exponencial doble que describe muy bien dicho el de difusión. Esta función proviene de datos recopilados en Guatemala entre 1990 y 1992 referentes al proceso de difusión de las dos variedades mejoradas de frijol, ICT A-Ostúa e ICT ASan Martín V.B. Se puede interpretar los dos parámetros de dicha función de la siguiente manera: el parámetro $a_{1}$ refleja la tasa marginal de substitución entre el rendimiento de la variedad mejorada y el rendimiento de la variedad del agricultor. El parámetro $\mathrm{a}_{2}$ se identifica como el cociente inverso de los costos de adquisición de las dos variedades de frijol, los mismos que se calculan con base al precio de las semillas y a los costos de transacciones. Este concepto permite darse cuenta de las dificultades que enfrenta el pequeño agricultor para conseguir semilla mejorada, tales como: información sobre cuándo y dónde procurarse semilla mejorada, costos de transporte, entre otros. La desventaja de esta función es que además de sobrestimar el número de los agricultores que adquieren semilla mejorada, en el año de la máxima demanda, sobrestima también el periodo de tiempo durante el cual se da la demanda. Un polinomio de tercer grado resultaría más apropiado, pero la interdependencia de sus parámetros hace difícil su interpretación. Este problema de interpretación no se presenta con la función exponencial doble.
\end{abstract}

\begin{abstract}
Diffusion process of improved bean (Phaseolus vulgaris L.) varieties in Guatemala. A double exponential function provides a good description of this diffusion process. This function was derived from the data concerning the diffusion process of the two improved varieties ICT A-Ostua and ICT ASan Martin V.B. in Guatemala in the years 1990-1992. The two relevant parameters may be interpreted in the following way: Parameter $\mathrm{a}_{1}$ reflects the marginal rate of substitution between the yields obtained from both, the improved and the farmer' $s$ bean varieties. Parameter $a_{2}$ considers the relationship of the acquisition costs among the two varieties, which are based on the seed price plus the transaction's costs. This concept allows to discern the difficulties faced by the farmers, such as the lack of information of where and when to acquire the improved seed, the delivery costs and so on. Unfortunately, this function no only tends to over-estimate the number of farmers who buy the improved seed during the year of maximum demand but al so the time span of this maximum demando A third degree polynome would be more appropiated, but its inter-dependent parameters are more difficult to interptet, in contrast to the easy interpretation of the double exponential function.
\end{abstract}

\section{INTRODUCCION}

En América Central, se empezó a discutir sobre sistemas de producción artesanal de semilla a partir de la segunda mitad de los años 80, aunque en Guatemala, hubo acciones de producción de semilla de frijol, por pequeños productores, desde 1973. En Guatemala, se organizó en
1987, en Jutiapa el Primer Taller Nacional de Producción Artesanal de Semilla (PAS). El PAS agrupó agricultores, extensionistas de DIGESA (Dirección General de Servicios Agrícolas) y técnicos de ICTA (Instituto de Ciencia y Tecnología). El taller fue organizado en Jutiapa en 1987, con la participación de profesionales del Centro Internacional de Agricultura Tropical (CIAT).

\footnotetext{
1 Presentado en la XXXIV Reunión Anual del PCCMCA, Guatemala, América Central. 28 de marzo - 3 de abril, 1993.

2 Ing. Agr. PROFIJOL-CIAT, Guatemala; Instituto de economía agrícola (1A W), ETH-Zentrum, CH-8092 Zürich.
} 
Se pueden agregar sistemas de PAS al sector no convencional ( $\mathrm{SnC}$ ). En cuanto a la modalidad de operación, en el $\mathrm{SnC}$ se distinguen dos modelos: el primero se refiere a productores individuales de semilla (PIS; Baltensweiler 1991) y el segundo a pequeñas empresas de semilla (PES; Garay 1991). Los sistemas de producción y comercialización de semilla del $\mathrm{SnC}$ puede oponerse al sector convencional (SC), el cual engloba el subsector privado y público. Otra denominación para el $\mathrm{SnC}$ es: sector informal en delimitación del sector formal. Los suministros de semilla, del SC se caracterizan por el control de calidad llevado a cabo por agencias de certificación. El objetivo de los nuevos suministros de semilla, en el esquema del $\mathrm{SnC}$, se orienta a aumentar la disponibilidad de variedades mejoradas destinadas a pequeños agricultores. La estructura descentralizada de los modelos P AS garantizan esta meta.

Para comprender el objetivo del presente trabajo, conviene aclarar dos puntos importantes:

1. La intensa autopolinización del Phaseolus vulgaris L., provoca que la oferta de semilla sólo encuentre comprador seguro en aquellos que la adquieren por primera vez. A los productos con estas propiedades se les conoce como "one-time purchased products" (Kloter 1984).

2. La composición de la clientela del SC y del SnC es diferente. Los clientes del $\mathrm{SnC}$ son pequeños agricultores orientados en general a la producción de subsistencia, lo que significa que la adquisición de una nueva variedad tiene como objetivo principal un cambio de la calidad genética. La renovación de la semilla para mejorar la calidad fisiológica, fitosanitaria y física es algo secundario (Baltensweiler 1993). La clientela del SC está compuesta por agricultores más grandes, quienes compran nueva semilla en periodos regulares de tiempo. A estos productos se les denomina "frequently purchased products" (Kloter 1984) en el ámbito de la comercialización.

La Figura 1, muestra el ciclo de vida de una nueva variedad en el SC y en el SnC. La primera adquisición de una nueva variedad se puede describir como un proceso de difusión que se caracteriza por un crecimiento muy lento al principio pero que viene seguido de un periodo de difusión de mayor velocidad. A la izquierda de la Figura 1 se muestra que este periodo se realiza entre el año 4 y 5 . El número de agricultores que requieren semilla llega después al máximo (indicado con la flecha horizontal al nivel de $\approx 20 \%$ ). Al final, la velocidad de difusión baja hasta cero y el proceso de difusión llegó a su término (indicado con una flecha en el año 7). Una adquisición frecuente, que hace que la demanda se estabilize, es el modelo adecuado para caracterizar la difusión de semilla en suministros del Se. A la derecha de la Figura 1, se muestra una adquisición frecuente en el año 3 y 6 (flechas verticales). Si se repite este proceso, la demanda de semilla se equilibra a un nivel distinto (flecha horizontal al nivel de $\approx 20 \%$ ).

Además, es un hecho que las escasas PES existentes en Centroamérica, se han formado basadas en el cultivo de frijol y limitadas a ese sector de explotación. Por ello, el riesgo económico de la empresa es más grande en comparación con empresas privadas o estatales del SC, quienes no se limitan solamente a ofrecer semilla de frijol. Esta argumentación demuestra que es de gran importancia entender el proceso de difusión, especialmente cuando la modalidad de operación se refiere al modelo de PES.

\section{MATERIALES Y MÉTODOS}

\section{Dos modelos de difusión}

En general, para describir el proceso de adopción se usa una función de distribución de forma $F(t)=\left(t, a_{1} \ldots a_{n}\right)$ donde $t$ es el tiempo y $\mathrm{a}_{1} \ldots \mathrm{a}_{\mathrm{n}}$ son los parámetros de la función $\mathrm{f}(\mathrm{t})$. La correspondiente función de densidad $\mathrm{f}(\mathrm{t})$ es la primera derivación de $\mathrm{F}(\mathrm{t})$ y representa la velocidad de difusión. El proceso inverso también es posible: si se sabe que $f(t):=g(t), G(t)$ se calcula para una integración de $g(t)$. Esta función de densidad se puede escribir de la siguiente manera: $g(t)=\left(t, b_{1} \ldots b_{n}\right)$ donde $t$ es el tiempo y $\mathrm{b}_{1} \ldots \mathrm{b}_{\mathrm{n}}$ son los parámetros de la función $\mathrm{g}(\mathrm{t})$. 

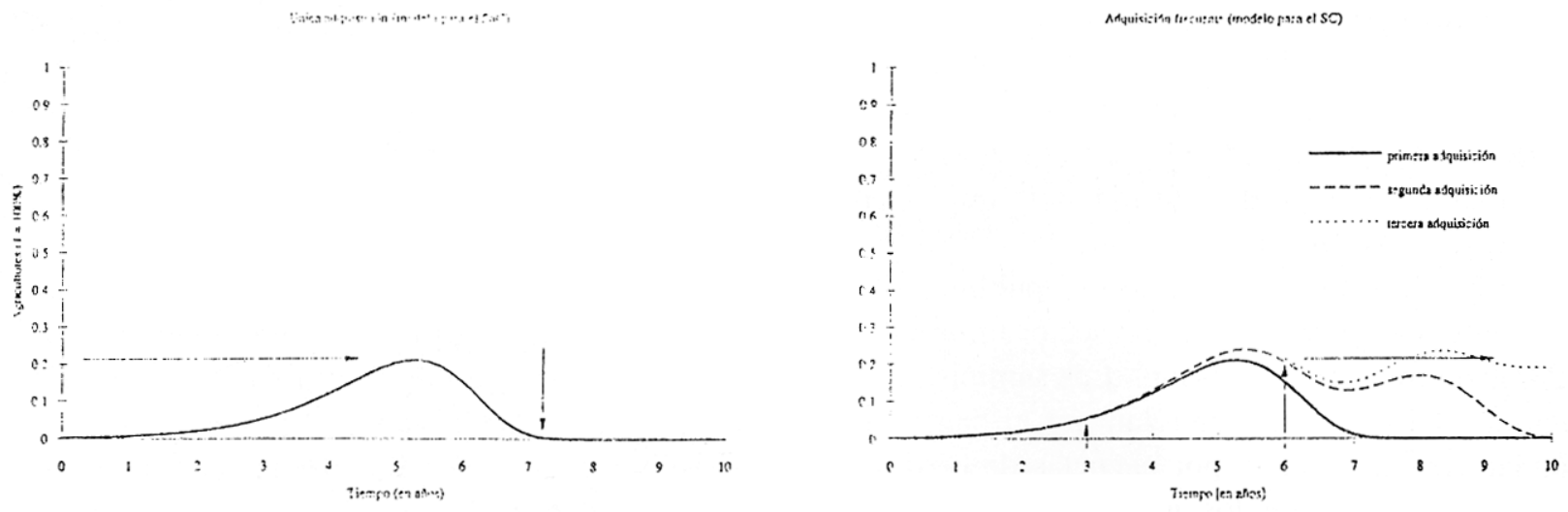

Figura 1: Ciclo de vida de variedades mejoradas en suministros de semilla del SnC y del SC (adaptado de Kloter 1984).

Las dos posibilidades han sido usadas para describir el proceso de difusión de las variedades ICTA-Ostúa e TCTA-San Martín V.B. Los dos procesos tienen como base diferentes funciones. La Fórmula I es una runción exponellcial doble y la Fórmula 2 representa un polinomio de tercer grado. Las dos fórmulas, 1a y $2 \mathrm{~b}$, son funciones relativas de distribución y las fórmulas lb y la representan funciones relativas de densidad.

Ambos modelos son discutidos en base a las experiencias de las variedades ICTA-Ostúa e ICTA-San Martín V.B. Los datos necesarios han sido recopilados mediante una encuesta representativa llevado a cabo en el año 1992. En el Departamento de Jutiapa se han entrevistado a 203 agricultores (con respecto a ICTA-Ostúa) y en el Departamento Chimaltenango se han hecho 197 encuestas (sobre la variedad ICTA-San Martín V.B.) con técnicos del servicio de extensión DIGESA. La pregunta era: "¿En qué año sembró usted por primera vez la variedad ICTA-Ostúa (y lo mismo para la variedad ICTA-San Martín V.B.)?"

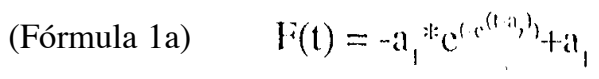

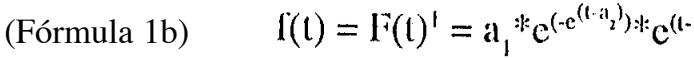

$$
\begin{aligned}
& \left.\mathrm{a}_{2}\right) * \operatorname{In}(\mathrm{c})^{2} \\
& \text { (Fórmula 2a) } \quad \mathrm{g}(\mathrm{l})=\mathrm{b}_{1}{ }^{*} \mathrm{t}^{3}+\mathrm{b}_{2}{ }^{* \mathrm{t}^{2}} \\
& \text { (Fórmula 2b) } \mathrm{G}(\mathrm{l})=\int \mathrm{g}(\mathrm{t})=1 / \mathrm{b}_{1} * \mathrm{t}^{\mathrm{i}}+1 / \mathrm{b}_{2} \mathrm{~b}^{*} \mathrm{t}^{3}
\end{aligned}
$$

\section{RESULTADOS}

La Figura 2 nos muestra los resultados. Se puede observar que el nivel de adopción de la variedad ICTA-Ostúa llega casi al 60 por ciento. La variedad ICTA-San Martín V.B. llega al 10 por ciento. Cabe preguntarse porqué la diferencia entre los dos ejemplos es tan grande. Antes de identificar los factores que influyen en los mecanismos de difusión, en el siguiente capítulo se discutirá sobre los dos modelos, usando los resultados de la variedad ICTA-Ostúa.

El R cuadrático es alto para los dos modelos (ver Cuadro 1). Desde este punto de vista ningún modelo puede favorecerse claramente. Óptico, el polinolTlio de tercer grado $\mathrm{g}(\mathrm{t})$ coincide mejor con las observaciones (= primera adquisición) en comparación con la primera derivación de $\mathrm{F}(\mathrm{t})$. 

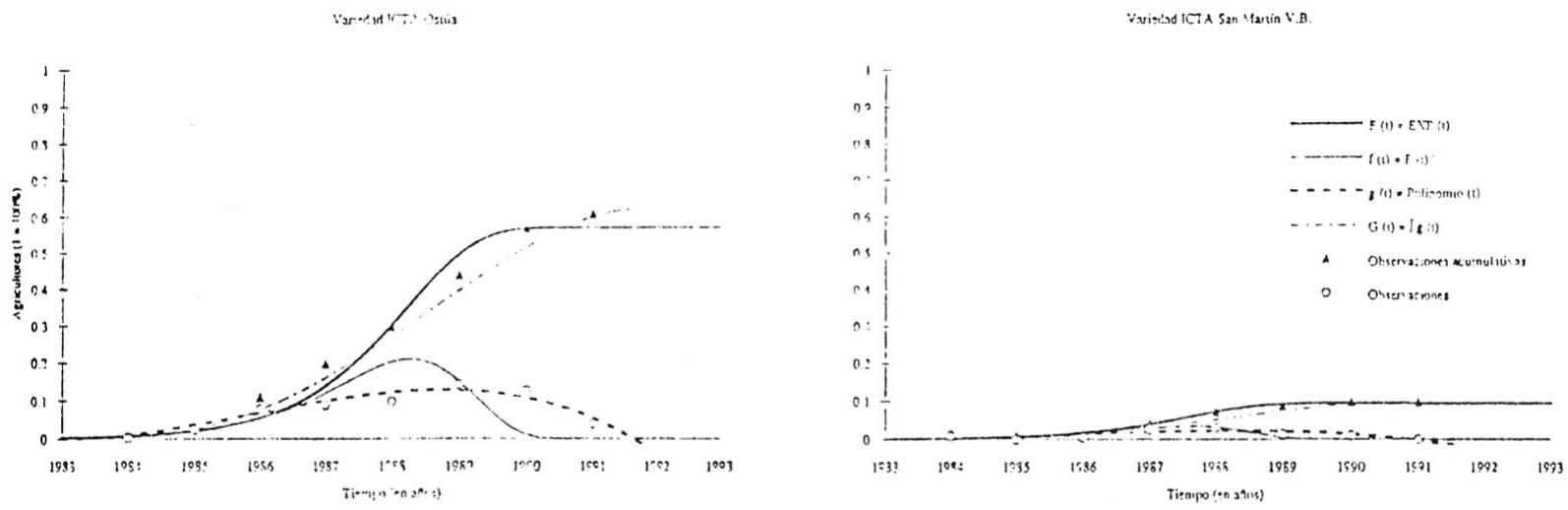

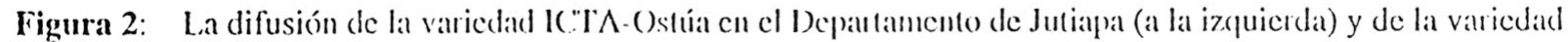
ICTA-San Martín V.B. cen el Depantamento de (himaltenango (a la derecha). Lstimación de las curvas según un modelo exponencial doble $|\mathrm{F}(\mathrm{t}), \mathrm{f}(\mathrm{l})|$ y según un modelo polinomio de tereer grado $\mid \mathrm{g}(\mathrm{t}), \mathrm{G}(\mathrm{t})]$.

Cuadro 1. Estimación de los parámetros.

\begin{tabular}{lcccccccc}
\hline & \multicolumn{3}{c}{$\mathbf{F}(\mathbf{t})$ Fórmula 1a } & & \multicolumn{3}{c}{$\mathbf{g}(\mathbf{t})$ Fórmula 2a } \\
\cline { 2 - 4 } \cline { 7 - 9 } Variedad & $\mathbf{a}_{\mathbf{1}}$ & $\mathbf{a}_{\mathbf{2}}$ & $\mathbf{R 2}$ & & $\mathbf{b}_{\mathbf{1}}$ & $\mathbf{b}_{\mathbf{2}}$ & $\mathbf{R 2}$ \\
\hline ICTA-Ostúa & 0,5702 & 5,284 & 0,989 & & $-0,001339$ & 0,01156 & 0,971 \\
ICTA-S. M. & 0,0939 & 4,662 & 0,996 & & $-0,000305$ & 0,00240 & 0,831
\end{tabular}

Los modos de las funciones $\mathrm{f}(\mathrm{t})$ y $\mathrm{g}(\mathrm{t})$ están como característica importante a la derecha de sus promedios. Este propiedad coincide muy bien con las observaciones y es la razón principal para no usar un modelo logístico como función de distribución. Las funciones logísticas son comunes para describir procesos de difusión. Su mayor desventaja para describir la difusión de variedades mejoradas es que sus modos coinciden con promedios (su función de densidad es a la vez una distribución normal). La diferencia entre la función $f(t)$ y $g(t)$ es que $f(t)$ es una función, extrema (generalmente usada para modelos de lluvias fuertes) y llega a un valor máximo más alto y en un periodo de tiempo más corto. Desde este punto de vista, resulta mejor usar el polinomio de tercero grado, teniendo en cuenta especialmente que para suministros de semilla del $\mathrm{SnC}$ es de suma importancia sincronizar la producción con la demanda de la primera adquisición. La desventaja de $\mathrm{g}(\mathrm{t})$ proviene de su sensibilidad de sus parámetros $b_{1} y_{b_{2}}$, teniendo como consecuencia que la aseguración estadística de los coeficientes de regresión no lineal $\left(b_{1}\right.$ y $\left.b_{2}\right)$ no sea grande. La utilidad del polinomio de tercero grado $\mathrm{g}(\mathrm{t})$ se ve, por un lado, en la justificación ue la demanda mfíxima, y por el otro, en la justificación del momento de llegada al máximo en comparación con el modelo exponencial $f(t)$. Como se ve en la Figura 2, la función $\mathrm{f}(\mathrm{t})$ sobrestima el nivel máximo de la primera adquisición y no representa bien el periodo de tiempo en que se llega a dicho máximo. 


\section{IDENTIFICACION DE LOS PARAMETROS y CONCLUSIONES}

En la siguiente parte, el objetivo es identifkar los factores que influyen en los mecanismos de difusión. En otras palabras, se busca la interpretación de los parámetros $a_{1}, a_{2}, b_{1}$ y $b_{2}$. Se puede identificar (1) factores que determinan principalmente el nivel de adopción y (2) factores que determinan la velocidad de difusión.

Identificación 1: Los factores que determinan el nivel de adopción son en general factores que dependen de la preferencia del agricultor. Por ello, el nivel de adopción de una nueva variedad liberada, se puede justificar únicamente en relación con las variedades cultivadas. En la siguiente argumentación se supone que los agricultores prefieren variedades con rendimientos altos. Desde este punto de vista, los factores abióticos, como el clima y bióticos, como la presencia de plagas y enfermedades que influyen los rendimientos, son de gran importancia. Como las variedades responden diferentemente a dichos factores abióticos y bióticos sus rendimientos difieren. El rendimiento de las variedades cultivadas (corrientes, $R_{c}$ ) y las nuevas variedades (mejoradas; $\mathrm{R}_{\mathrm{m}}$ ) determinan principalmente pero no exclusivamente el nivel de adopción. En la teoría de producción, esta relación es conocido como tasa marginal de substitución [TMS $=\Delta \mathrm{R}_{\mathrm{m}} / \Delta \mathrm{R}_{\mathrm{c}}$ ].

Las experiencias llevadas a cabo de ensayos en fincas en los Departamentos de Jutiapa y de Chimaltenango confirman esta afirmación: En Jutiapa, el rendimiento de las variedades criollas llega a un promedio de 660 kilogramos por hectárea comparado con los 1260 kilogramos por hectárea de rendimiento de la variedad ICT A-Ostúa. En Chima]tenango, el rendimiento de la variedad ICT A-San Martín V.B. $(620 \mathrm{~kg} / \mathrm{ha})$ no es significativamente superior a las criollas (570 kg/ha) (Baltensweiler 1992). Los datos son comprensible si se tiene en cuenta que en el Departamento de Jutiapa el mosaico dorado (BGMV) es considerado el problema principal.
Conclusión No 1: La relación funcional entre el nivel de adopción y el rendimiento de las diferentes variedades reza en su forma más simple: si la tasa marginal de substitución (TMS) de las variedades es elevada, la adquisición acumulativa de la variedad mejorada llega a un nivel de adopción alto $\left(\mathrm{n}_{\mathrm{ad}}\right)$ [si TMS $\neq \mathrm{fin}_{\mathrm{ad}} \neq$ ] .

Según una sustentación controvertida, se puede afirmar que el nivel de adopción no depende del sistema de extensión ni de promoción.

Identificación No 2: La velocidad de difusión se determina (1) ségun los factores socio-económicos de la clientela y (2) ségun la eficiencia del sistema de producción y promoción de parte de la oferta. En otras palabras: los factores socio-económicos y la eficiencia del suministro de semilla determinan también, principalmente pero no exclusivamente, el periodo de tiempo en el cual una variedad llega su nivel máximo de adopción.

Los factores socio-económicos de la clientela pueden ser: la liquidez y los costos de los transacciones que se originan cuando el agricultor busca semilla de una nueva variedad, etc. De Janvry y otros, 1992 definen como costos de transacciones los costos que provienen de informaciones erróneas, búsqueda, regateo, transporte etc..

El factor más adecuado para medir la eficiencia de cualquier sistema de producción es todavía, según ]a teoría neoclásica, el precio del producto. El concepto de los costos de transacciones permite integrar los costos de adquisición en una sola variable. Permite también desechar el concepto fijo de que el precio es la única variable que determina el punto equilibro entre la oferta y la demanda: los costos de adquisición $\left(\mathrm{c}_{\mathrm{ad}}\right)$ equivalen al precio de la semilla $\left(\mathrm{p}_{\mathrm{s}}\right)$ mas los costos de transacciones $\left(\mathrm{c}_{\mathrm{tr}}\right)$ por un kilogramo de semilla $\left[\mathrm{c}_{\mathrm{ad}}=\mathrm{P}_{\mathrm{s}}+\mathrm{c}_{\mathrm{tr}}\right.$ ). La teoría de producción usa el cociente inverso de los precios de los factores para determinar la combinación óptima de dos factores. Aplicando ésto a los costos de adquisición tenemos que el cociente inverso de adquisición (CIA) se da entre los cos- 
tos de adquisición de las nuevas variedades (mejoradas; $\mathrm{c}_{\mathrm{adm}}$ ) y los costos de adquisición de variedades cultivadas (corrientes, $\mathrm{C}_{\mathrm{adc}}$ ) $\left[\mathrm{CIA}=\mathrm{c}_{\mathrm{adc}} / \mathrm{c}_{\mathrm{adm}}\right]$.

Conclusión No 2: La relación funcional entre la velocidad de difusión y el cociente inverso de los costos dc adquisición reza, cn su forma más simplc: si el cociente inverso de los costos de adquisición de semilla (CIA) es bajo, la demanda máxima de la primera adquisición (respectivo el modo de la primera adquisición), se realiza $\sim \mathrm{n}$ un periodo de tiempo corto $\left(\mathrm{t}_{\mathrm{mo}}\right)$ [si CIA $\varnothing$ fi $\mathrm{t}_{\mathrm{mn} \emptyset}$ ].

Ségun una sustentación controvertida, se puede afirmar que el periodo, en el cual la primera adquisición llega a su máximo nivel, no depende de factores bióticos y abióticos.

Las relaciones funcionales mencionadas en la conclusión 1 y 2 deben ser especificadas, pero ello no constituye el objetivo del presente artículo.

Las conclusiones 1 y 2 están formuladas estrictamente, de manera que el nivel de adopción es independiente del periodo en el cual se alcanza dicho nivel máximo. Las condiciones mencionadas en las identificaciones 1 y 2 aseguran que el nivel de adopción y el periodo en el que ésta se realiza son interdependientes. Por ello, tales condiciones parecen ser más realistas que las dos estrictas conclusiones. El modelo del polinomio representa estas condiciones, pero como la interpretación de los parámetros $b_{1}$ y $b_{2}$ resulta difícil. Este punto no es tratado más a fondo.

Como el parámetro al de la función exponencial determina el nivel de adopción, la interpretación de al se refiere a la conclusión No 1: $a_{1}$ es una función de la tasa marginal de substitución entre la nueva variedad y las variedades corrientes $\left[\mathrm{a}_{1}=\mathrm{n}_{\mathrm{ad}}=\mathrm{f}(\mathrm{TMS})\right]$.

El parámetro $a_{2}$ de la función $\mathrm{f}(\mathrm{t})$ determina el periodo en el que aparece el modo. Tomando en cuenta la conclusión No 2, a $a_{2}$ es una función del cociente inverso de los costos de adquisición $\left[\mathrm{a}_{2}=\mathrm{t}_{\mathrm{mo}}=\mathrm{f}(\mathrm{CIA})\right]$.

En la Figura 3 se pueden visualizar estos conceptos. Como se mencionó en la introducción, los suministros alternalivos de semilla del SnC tienen como objetivo de aumentar la disponibilidad de variedades mejoradas. A la izquierda de la Figura 3 se ve claramente que las modalidades del PAS pueden acelerar el proceso de difusión (desplazamiento de las curvas hacia la izquierda).
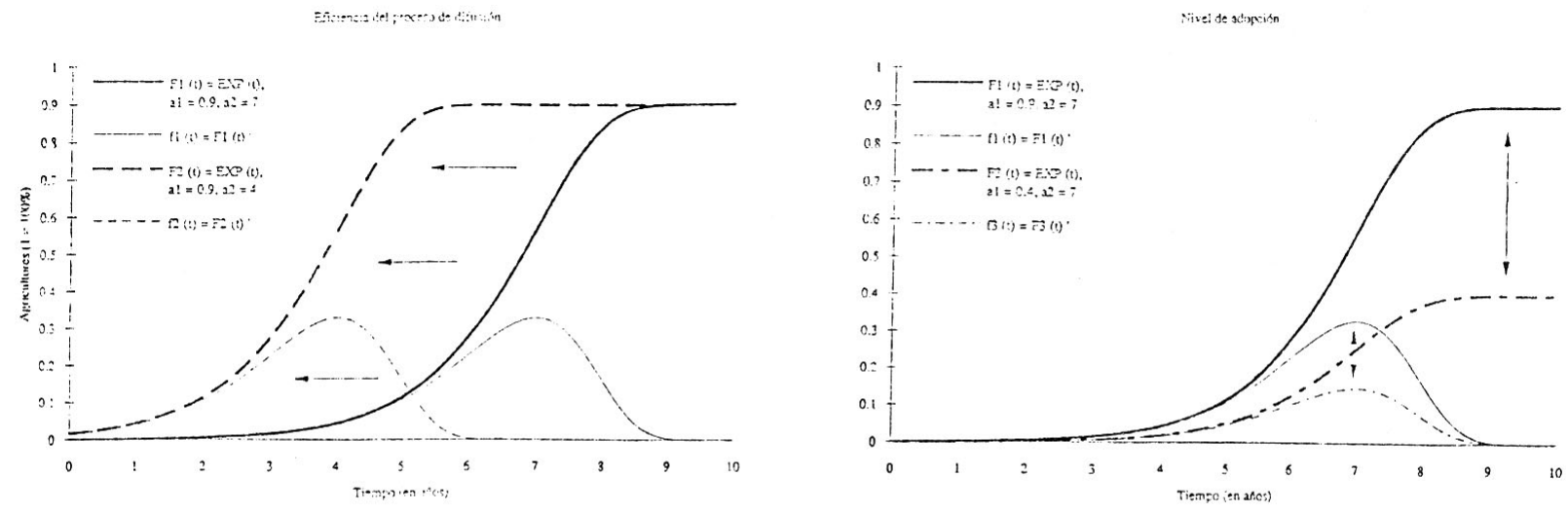

Figura 3: La eficiencia del proceso de difusión y el nivel de adopción en base de diferentes valores de los parámetros de la función exponencial doble $a_{1} y^{\prime} a_{2}\left(a_{1}=n_{a j} ; a_{2}=t_{m o}\right)$. 
El éxito radica en la disminución de los costos de adquisición de semilla por parte de la decentralización de los suministros de semilla. En este contexto, se pueden mencionar dos puntos de interés práctico: el primero se refiere a la experiencia con una PES, y el segundo a la modalidad de PIS, ambos en el Departamento de Jutiapa. Las dos experiencias negativas se basen en el hecho de que el proceso de difusión, como fue presentado con el modelo exponencial doble, no fue tomado en cuenta durante la realización de los proyectos.

1. La PES Santa Gertrudis ha fijado el precio de su semilla casi al mismo nivel en el que el SC ha ofrecido su semilla. La ventaja comparativa de la PES se resumía casi a los costos de transacciones, que eran incluso más bajos para el agricultor, comparado al costo de un viaje a la capital donde se vende la semilla del Se. Desde este punto de vista, la eficiencia del proceso de difusión mediante a la PES Santa Gertrudis puede set mejorada.

2. En el año 1992, la agencia de DIGESA en Jutiapa adoptó la modalidad PIS de producción de semilla. La producción total era suficiente para siembra en la época siguiente con la variedad ICT A-Ostúa en más de 300 hectáreas. En realidad, las 300 hectáreas sólo representan el 1.2 por ciento del área total cultivada con frijol en el Departamento de Jutiapa. La crítica es que el proceso de difusión se terminó en el año 1991, como lo muestra la Figura 2. Los PIS estaban obligados a vender su cosecha de semilla como granos o a buscar agricultores que renueven su semilla. La existencia de un mercado para adquisiciones frecuentes aún no está activa o asegurada.

A la derecha de la Figura 3 se muestra que el nivel de adopción determina el volumen total de semilla requerida en el año. Un nivel de adpoción potencial de 90 por ciento provoca que 30 por ciento de los agricultores aquieren la variedad en el año de la damanda máxima. Si el nivel de adpoción potencial es tan sólo del 40 por ciento, la demanda máxima llegará en ese mismo año a solamente el 10 por ciento.
En sus planes de producción, los suministros de semilla deben de tomar en cuenta el proceso de difusión corpo acaba de ser presentado. La demanda se puede estabilizar únicamente, si existe una regularidad en la adquisición frecuente de semilla en la región.

\section{LITERATURA CITADA}

BALTENSWEILER, M. 1991. Los tres suministros de Semilla de Frijol en Guatemala. Aspectos de comercialización de semilla en el suministro no convencional. Curso de Producción artesanal de semilla. DIGESA / ZACAP A. 24. 26. de Septiembre 1991, Zacapa. (no publicado)

BALTENSWEILER, M. 1992. Resultados sobre el estudio de los tres sistemas de suministro de Semilla de Frijol en Guatemala. Taller PROFRIJOL / CIAT. 2. - 3. de Junio 1992, Guatemala. (no publicado)

BALTENSWEILER, M. 1993. Los tres sistemas de suministro de semilla de frijol (Phaseolus vulgaris L.) en Guatemala. ¿Cuál sistema cumple en mejor forma con las necesidades del agricultor de un determinado grupo meta? Estudio de caso realizado en los departamentos de Jutiapa y Chimaltenango. Documento de Trabajo No 93 PROFRIJOL / CIAT Guatemala, Guatemala. (en preparación)

GARAY, A. 1991. Consideraciones claves para el desarrollo de pequeñas Empresas de semillas. En: Memorias. Taller centroamericano sobre desarrollo de pequeñas empresas de semilla. 22-26 de Abril 1991, Jutiapa, Guatemala. PRO FRIJOL, San José, Costa Rica. pp. 11.

DE JANVRY, A.; SADOULETLET, E.1992. Structural Adjustment under Transactions Costs. In: 29 th European Association of Agricultural Economists (EAAE) meeting on Food and Agricultural Policies under Structural Adjustment, 1992, Hohenheim, p. 101 - 126.

KOTLER, P. 1984. Marketing Management: Analysis, Planning and Control. Prentice Hall International Inc. London. 